\title{
SPINO-CORTICAL FIBRES IN MAN
}

\author{
BY \\ P. W. NATHAN and MARION C. SMITH \\ From the Neurological Research Unit, National Hospital, Queen Square, London
}

The purpose of this paper is to present evidence that in man there is a tract which originates in the spinal cord, ascends in the medulla and pons with the cortico-spinal tract, and runs through the internal capsule towards the cerebral cortex. It is also our purpose to draw attention to the older literature on this subject, and to review the evidence presented there.

In 1893 Sottas published a short report entitled " On Retrograde Degeneration of the Pyramidal Tract ", in which he described four cases of syphilitic softening of the thoracic region of the cord. In these cases he found degeneration cranial to the lesion in the region of the lateral cortico-spinal tract, which he traced into the cervical cord. In 1894 Gombault and Philippe reported similar findings in four cases. Raymond (1894) also reported a case of syphilitic transverse myelitis; he found, cranial to the lesion in the upper thoracic cord, "considerable diminution of myelinated fibres" and sclerosis in the region of the lateral cortico-spinal tracts ; these changes faded cranially, and could not be seen above the third cervical segment ; the region of the ventral cortico-spinal tracts was normal. In 1895 Egger found in a case of compression of the cord degeneration in the ventral and lateral cortico-spinal tracts cranial to the lesion; he considered that these were short intersegmental fibres. In 1896 Dejerine and Sottas reported a case of transverse myelitis due to syphilis ; they traced degeneration in the region of the lateral and anterior cortico-spinal tracts up into the decussation of the pyramids. In the same year, Dercum and Spiller reported a case of syringomyelia with " ascending (cellulipetal, retrograde)" degeneration involving the lateral and ventral corticospinal tract on one side. The most cranial part of the lesion was in the mid-thoracic region. The degeneration could be traced in the lateral corticospinal region to the second cervical segment and in the ventral cortico-spinal region to the pyramidal decussation. In 1898 Spiller reported another case. Further cases have been reported by Worotynski (1897), Stewart (1901), Petrén (1903), and Ramsay
Hunt (1904). In 1905 Bing reported the case of an infant with anterior poliomyelitis, affecting particularly the upper lumbar segments of the cord. The regions of the anterior and lateral cortico-spinal tracts were full of degeneration granules. From the nature of the pathology Bing had no doubt that these degenerating fibres originated in the grey matter of the cord. He concluded from the position of the degenerating axons that the cells of origin of the ascending fibres within the region of the lateral cortico-spinal tract were situated in the anterolateral part of the posterior horns and the cells of origin of the ascending fibres within the region of the ventral cortico-spinal tract were in the medial part of the anterior horns. Those in the ventral cortico-spinal tract could be traced cranially throughout the length of the cord; from their position abutting against the median fissure, Bing reckoned them to be part of Marie's faisceau sulco-marginal ascendant. He could not trace the fibres within the lateral cortico-spinal tract above the twelfth thoracic segment. He referred to this case again in his monograph on the spino-cerebellar system (1907), stating that these are ascending endogenous fibres "which can be followed several segments upwards".

From surveying this literature, it is clear that the workers who first described these degenerating fibres never considered that they might be dealing with a true spino-cortical tract. Sottas, Gombault and Philippe, Raymond, Dejerine and Sottas, Dercum and Spiller, Spiller, and Ramsay Hunt, all assumed that this was an example of retrograde degeneration. Gombault and Philippe, however, admitted that it was difficult to distinguish this form of degeneration from Wallerian degeneration. The belief that these fibres are descending fibres showing retrograde degeneration was not held by Egger or by Bing. In that they postulated that these fibres are short intersegmental fibres, they tacitly accepted that they were dealing with true Wallerian and not retrograde degeneration.

The first worker to state that he saw no reason for regarding these fibres as being other than a true 
spino-cortical tract, showing Wallerian degeneration, was Choroschko (1909). He reported two cases of compression of the cord in which degeneration could be traced through the pyramidal decussation and the internal capsule into the corona radiata. $\mathrm{He}$ gave detailed descriptions of the fibres, but he did not state his staining methods, nor did he give any drawings or photographs. He not only proposed that this was a true spino-cortical tract, analagous to the cortico-spinal tract, but he wrote that there never had been any more reason to consider retrograde degeneration in the case of this tract than in the case of any other divided tracts.

All these earlier observations seem to have been forgotten. Tower (1944) for instance wrote : "All that can be said with certainty is that all fibres running lengthwise in the medullary pyramids are descending fibres ". And Brodal and Walberg (1952): "It was surprising to discover in an experimental cat material prepared for other purposes that there are, in fact, ascending fibres in the pyramid and that such fibres take origin in the spinal cord". And later in the same paper, they wrote : "It may seem strange that these fibres, as far as we know, have not been observed by previous authors."

Brodal and Walberg's work is particularly important, for they not only showed degeneration in these fibres in the cat by means of the Marchi method, but they also showed it in serial sections stained by Glees' silver method. They were able to follow their ascending fibres into the sixth layer of the cortex : "They could be followed in decreasing numbers to the second layer, the external granular lamina." The area concerned was "the anterior part of the posterior sigmoid gyrus"; this is the region containing the giant pyramidal (Betz) cells. They observed that the cervical component of this ascending tract " appears to be relatively most important, since there is a pronounced difference between the results of high cervical and those of mid-thoracic lesions", and they found that "the more caudal the lesion, the more moderate the number of degenerating fibres ".

During the past seven years, while engaged on work on the anatomy of the human spinal cord, we have searched for ascending degeneration in the region of the cortico-spinal tracts. We have come to the conclusion that this degeneration is due to degeneration of a true ascending tract, which takes its origin within the spinal cord and runs, presumably, to cortical cells. This tract follows the path of the descending cortico-spinal tract as far as the cerebral peduncles; cranial to the pons, in the peduncle, and in the internal capsule the tract lies posterior to the cortico-spinal tract ; here its fibres lie among those of the temporo-pontine tract. The evidence for these statements will now be presented.

\section{Material}

Our material comes from patients who had the operation of spino-thalamic cordotomy for the relief of intractable pain. In this operation, fibres of the spino-thalamic, spino-cerebellar, spino-reticular, and spino-tectal tracts are divided. In the majority of these patients, the lesion involved not only these ascending fibres but also some descending fibres lying in, or near, the lateral cortico-spinal tract.

Forty-three patients are considered here ; they are divided into two groups. In the first group are eight patients in whom the operation was performed at the second or third cervical segments. Of this group, three patients died too soon after the operation (six hours, two days, and five days) and one patient too late (313 days) for the application of histological techniques which demonstrate the course of a few degenerating fibres. In the other four patients (Cases 37, 20, 35, and 39) the periods of survival were $81,110,112$, and 185 days respectively. In the second group are 35 patients in whom the operation was performed at various levels between the fifth cervical and first lumbar segments. In this group the interval between operation and death varied between 12 and 468 days. The evidence for the existence of a spino-cortical tract comes mainly from the first group, and so these cases will be considered in detail ; the cases of the second group provide supporting evidence.

Marchi preparations and/or cell and fibre preparations were made of all levels of the cord and brain, excluding the corona radiata and cortex.

\section{Observations}

Case 37 will be taken as an example of the degeneration found in the four cervical cases.

Operation Level (Fig. 1).-The operation was performed at the third cervical segment. The lesion caused by the operation extended dorsally to

All photographs are from Marchi preparations. Degenerating fibres are stained black.

Figs. 1 to 6 are all of Case 37. The interval between the lesion and death was 81 days.

FIG. 1.-Third cervical segment, showing the extent of the lesion caused by operation.

FIG. 2.-First cervical segment, showing degenerating fibres in the cortico-spinal tract cranial to the lesion.

FIG. 3.-Upper limits of pyramidal decussation, showing degenerating fibres in the decussation and in the pyramids, especially on the right, contralateral to the lesion.

Fig. 4.-Pyramids, showing scattered degenerating fibres, more numerous on the right.

FIG. 5.-Cortico-spinal bundles in the pons, with scattered degenerating fibres.

Frg. 6.-The right internal capsule, showing concentration of degenerating fibres in the posterior part. 


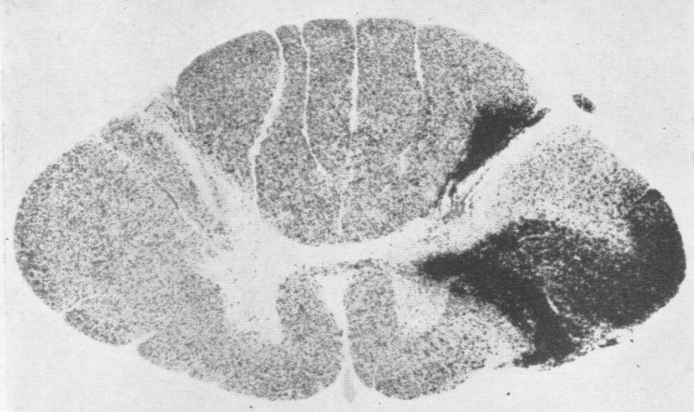

FIG. 1

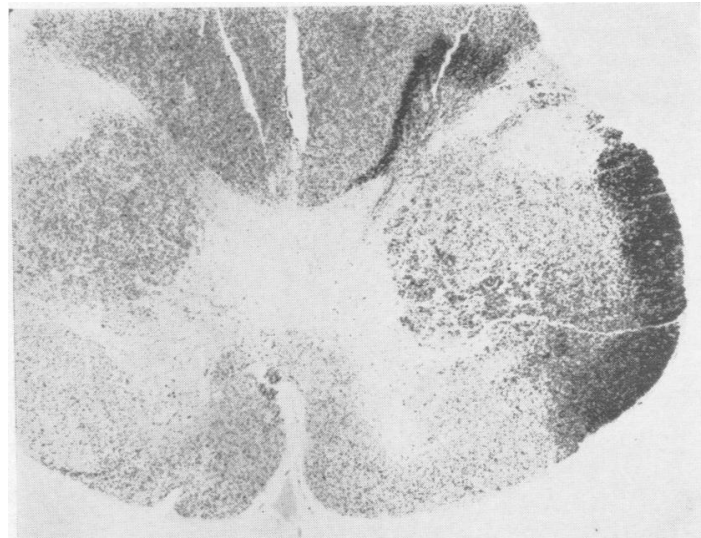

FIG. 2

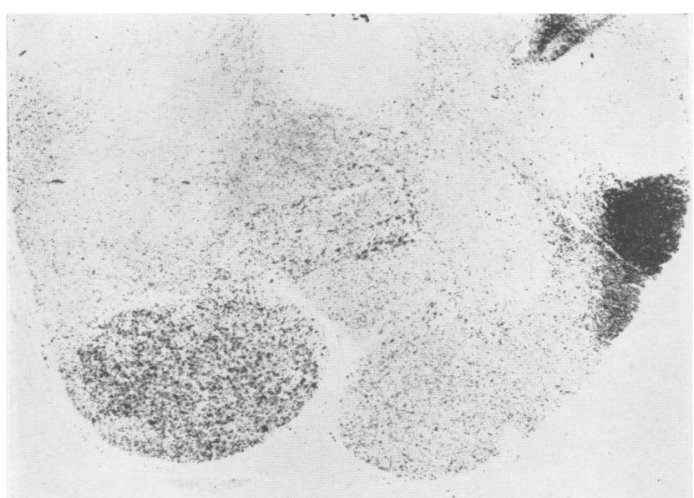

FIG. 3

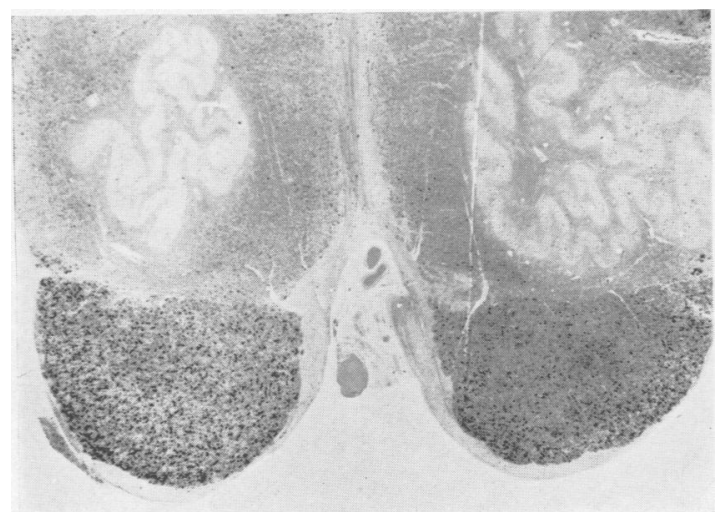

FIG. 4
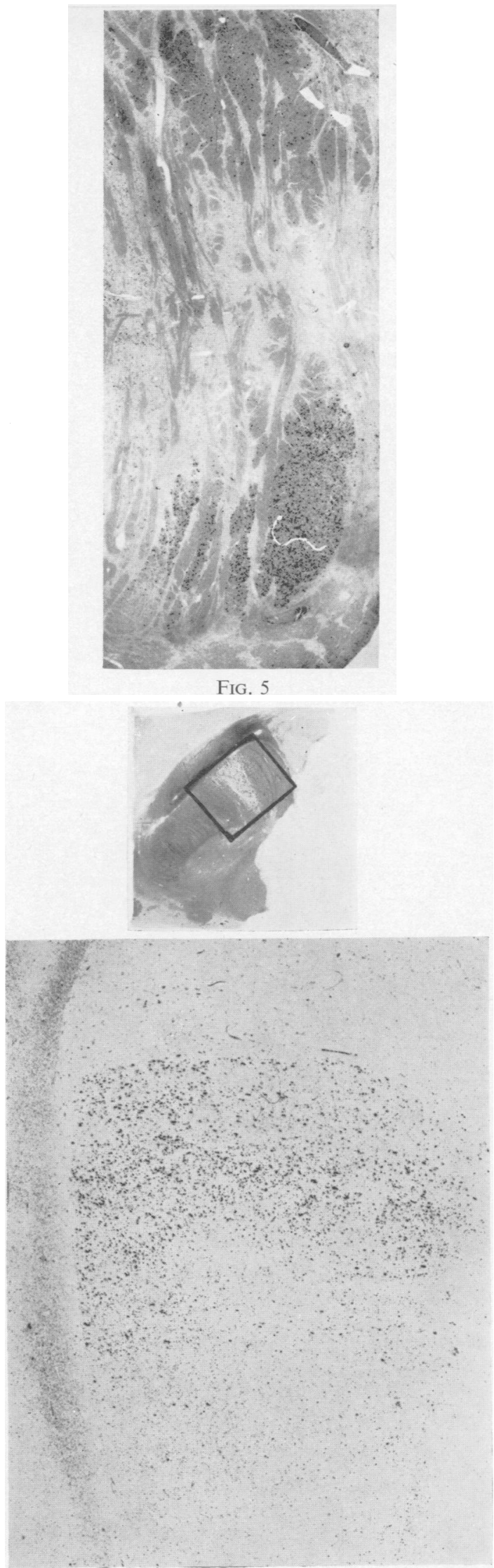

FIGs. $6 \mathrm{a}$ and $6 \mathrm{~b}$

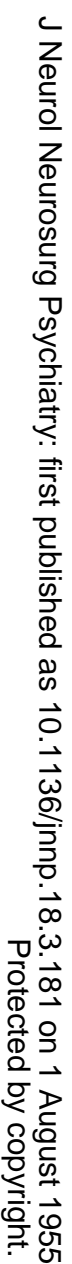

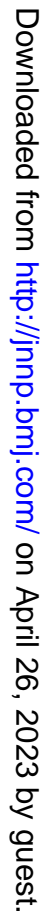




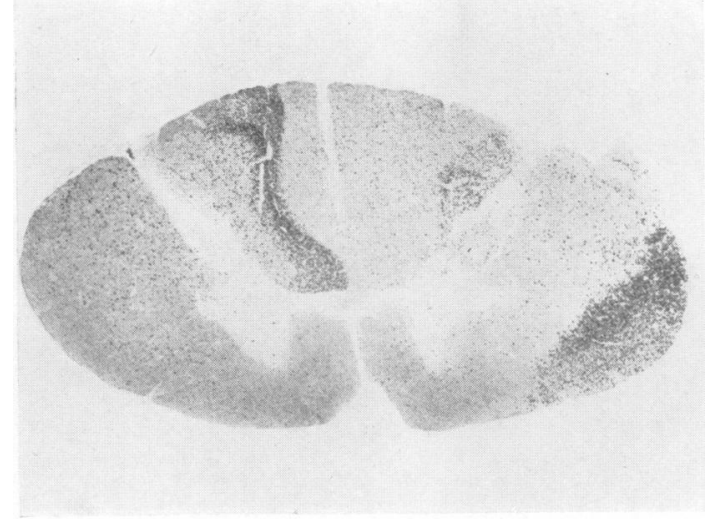

Figs. 7, 8, and 9 are from Case 35 . The interval between the lesion and death was 112 days.

FIG. 7.-Third cervical segment, showing the extent of the lesion caused by the operation on the left, and a small area of softening on the right.

Fig. 8.-Pyramidal decussation showing degenerating fibres from both sides of the cord.

Fig. 9.- Internal capsule at the dorsal limit of the thalamus, showing degenerating fibres in the retro-lenticular part.

FIG. 7

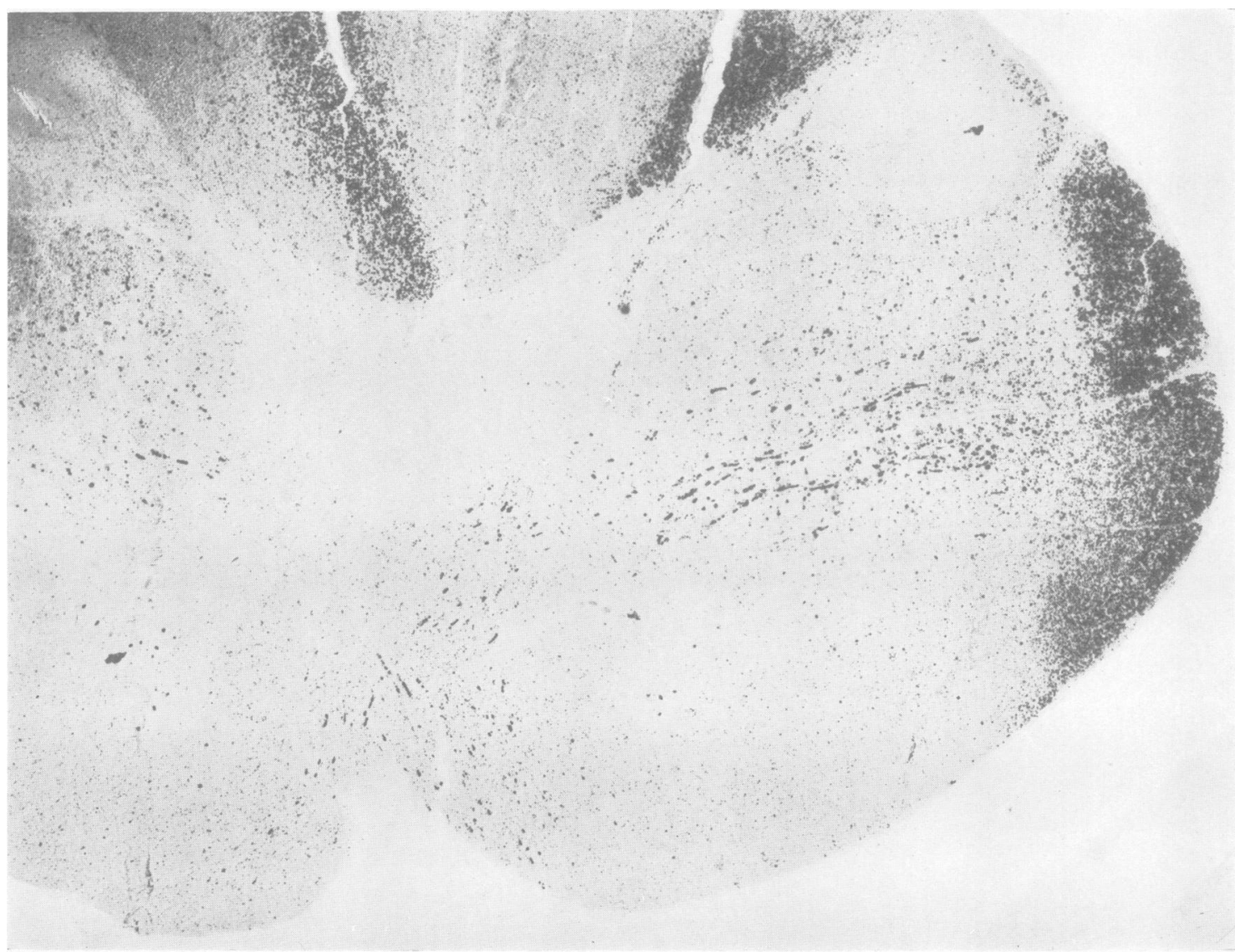

Fig. 8 


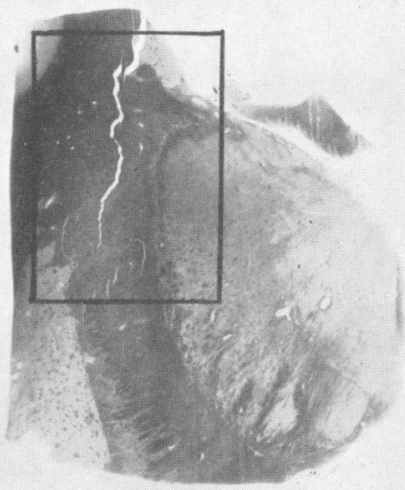

FIG. 9a

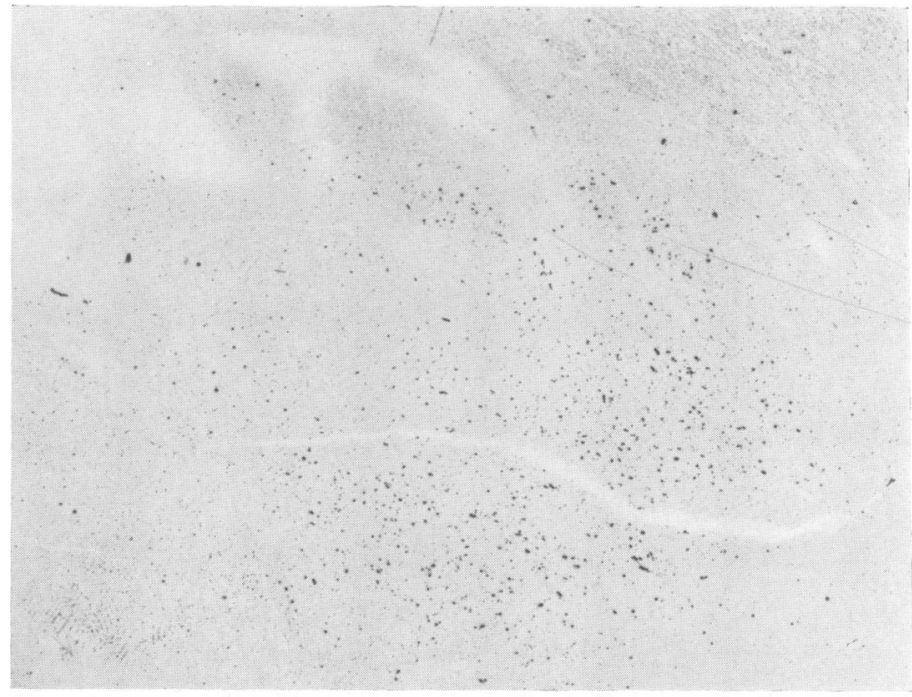

FIG. 9c 
Figs. 10 and 11 are from Case 39. The interval between the lesion and death was 185 days.

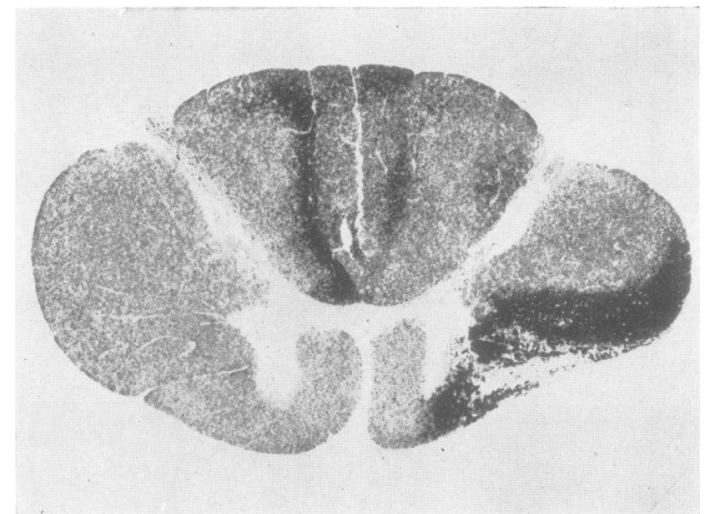

FIG. 10.-Second cervical segment showing the extent of the lesion caused by the operation.

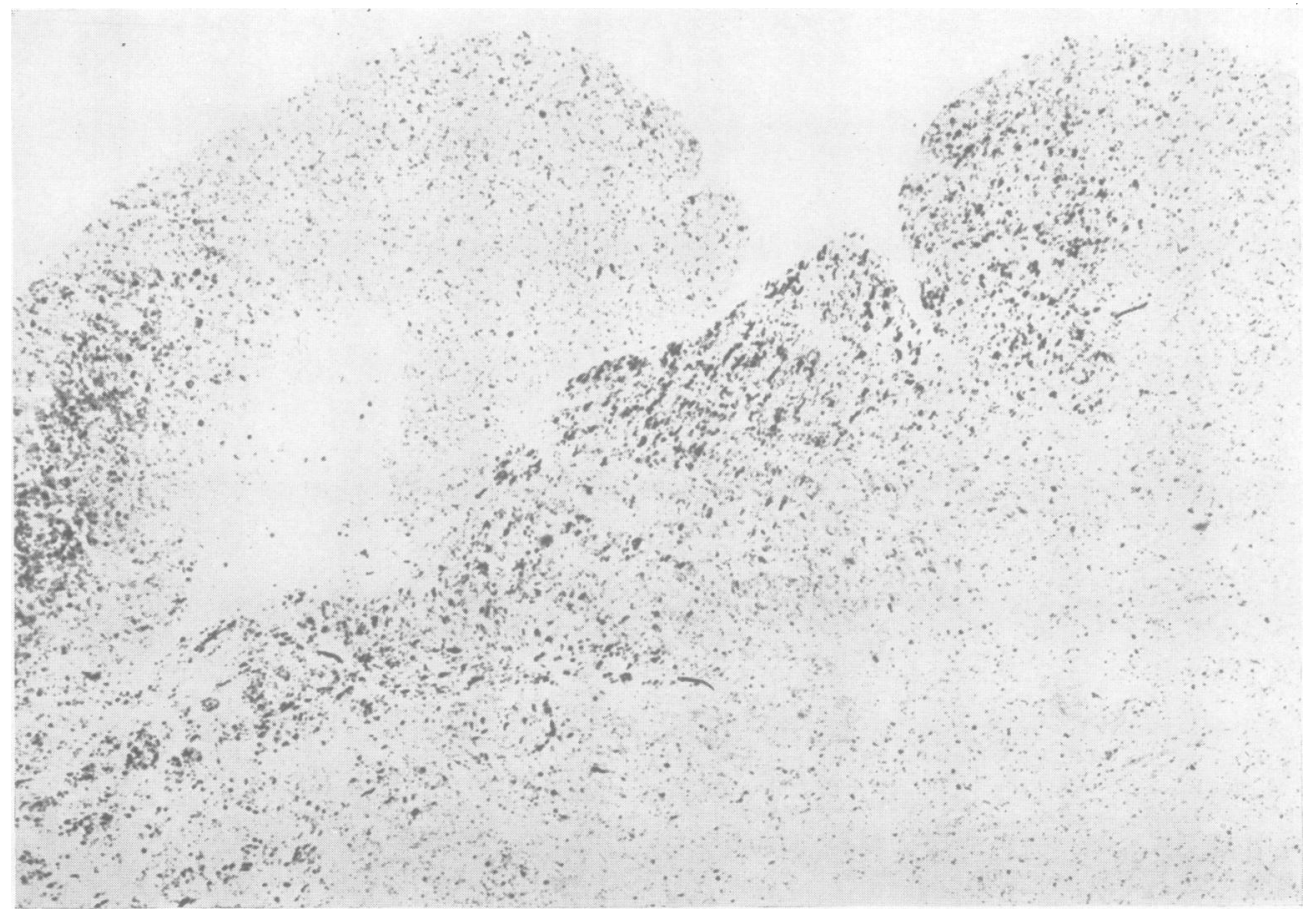

Fig. 11.-Pyramidal decussation showing degenerating fibres passing to the contralateral pyramid. 
approximately midway between the denticulate ligament and the posterior horn; it extended ventrally to slightly anterior to the ventro-lateral sulcus, and it extended medially into the grey matter of the cord.

First Cervical Segment (Fig. 2).-Degenerating fibres are present in the ventral part of the lateral cortico-spinal tract. In addition, degeneration is present in the spino-cerebellar, spino-thalamic, and associated tracts.

Upper Level of Pyramidal Decussation (Fig. 3). -Degenerating fibres can be seen crossing in the pyramidal decussation and also scattered throughout the pyramids. The greater number are in the right pyramid, contralateral to the lesion.

Pyramids (Fig. 4).-As in the previous section, the degenerating fibres are scattered diffusely throughout the pyramids, mainly on the right side. There is considerable variation in the size of the Marchi bodies, many being large, comparable to the degenerating fibres of the dorsal spino-cerebellar tract.

Pons (Fig. 5).- The degenerating fibres here, as in the pyramids, are diffusely scattered through the bundles of descending fibres ; the majority of them are on the right side. There appear to be fewer degenerating fibres here than in the pyramids.

Internal Capsule (Fig. 6).- - Here the degenerating fibres are concentrated in a small area in the posterior part of the internal capsule. This region is that occupied by the temporo-pontine tract. There are definitely fewer degenerating fibres here than in the more caudal sections.

In Case 35 (Figs. 7, 8, and 9) there were two lesions in the spinal cord. In addition to the surgical lesion made on the left side of the cord at the level of the third cervical segment, there was a small area of softening on the right side at the same level, involving the dorso-lateral region (Fig. 7). Degenerating fibres can be traced cranially from both these lesions. In Fig. 8 they are shown in the pyramidal decussation. In Fig. 9 they are shown in a horizontal section of the internal capsule at the level of the dorsal limit of the thalamus. It is to be noted that there is a considerable number of these fibres still present at this level, although the lesion was not extensive and the period of survival was long (112 days). In Case 39 a particularly large number of degenerating fibres is present. The extent of the lesion in the cord is shown in Fig. 10 ; it will be noted that in this case it was extensive. Numerous degenerating fibres in the pyramidal decussation are shown in Fig. 11. As Case 20 is similar in all respects to Cases 35,37 , and 39 , photographs of this case are not shown here.

It is to be stressed that in none of the four cases described here was there any evidence of a lesion of any part of the cortical-spinal system occurring at levels cranial to the operation. As serial sections of the material were studied, even a small lesion would certainly have been detected.

Conclusions from Histological Findings. - In all the cases with lesions in the upper cervical cord, the lesions extended sufficiently far posteriorly to cause clear descending degeneration in, or adjacent to, the lateral cortico-spinal tract. In all these cases there are some ascending degenerating fibres among the intact fibres of the cortico-spinal tract.

It has not been possible to determine the cells of origin of this tract, for the lesion in each case divided the fibres of many ascending systems as well as these fibres. We have mapped out the location of these cells, caudal to the lesion, which show retrograde changes. These cells are in various nuclei of the posterior and anterior horns and of the intermediate grey matter. It is impossible in our cases to determine which of these cells gave origin to the degenerating fibres under discussion.

The course of these spino-cortical fibres is as follows. Most, but not all of them, cross in the pyramidal decussation. They are diffusely scattered in the pyramids and in the cortico-spinal tract area of the pons; further cranially they become concentrated into a small region of the crus and of the posterior part of the internal capsule. In the highest levels of the internal capsule they are confined to the retro-lenticular area. Thus in the capsule their course is not the same as that of the descending cortico-spinal fibres, for they lie posterior to the cortico-spinal tract in the region of the temporo-pontine tract. It seems that many of these ascending fibres must go to the cortex, for there is no other grey matter at this level. It is clear from the fact that there is a gradual diminution in the number of these fibres as they course cranially that not all of them run to the cortex, or even as high as the thalamus, but the end-stations of these shorter fibres have not so far been determined.

It will be remembered that the lesion in our cases was limited to the anterior part of the lateral cortico-spinal tract. It is possible that fibres from the entire cross-sectional area of the lateral corticospinal tract do not all go into the retro-lenticular part of the internal capsule.

As has been stated, these fibres were most numerous when the lesion was in the upper cervical cord. Our material does not permit conclusions to be drawn about such fibres arising in the segments 
between the third cervical and the first thoracic segment, for in this region we have only two cases, and in both of them the lesion was restricted to the antero-lateral and anterior parts of the cord. We have many cases with more caudal lesions than the first thoracic segment; in these only a few fibres could be traced cranially. From this material it is concluded that the majority of these fibres originate in the cervical region.

If these fibres followed the entire course of the cortico-spinal tract, the question of whether the degeneration which they show is an example of Wallerian degeneration or of retrograde degeneration would be all-important. But as they leave the course of the cortico-spinal tract cranial to the pons they must be a distinctive tract. In view, however, of the reports of the original workers on this subject, it is to be stated that the features of the degeneration in this tract are exactly the same as those of the Wallerian degeneration in all other damaged tracts; in every case, the stage and appearance of the degeneration was identical with that of the spino-thalamic, spino-cerebellar, and cortico-spinal tracts.

The Marchi bodies vary in diameter from 3 to $25 \mu$, with many in the 15 to $20 \mu$ range. As Marchi bodies represent disrupted myelin sheaths, they cannot be taken as indicating the exact calibre of the fibres from which they came. It would be particularly fallacious to take the smaller bodies as representing only fine fibres for they may represent degenerating myelin from thicker fibres which have broken up. Nevertheless some deductions from the size of the larger Marchi bodies may be made. We have observed that large Marchi bodies occur only in tracts known to contain thick fibres. And so, although we cannot draw conclusions about the fibre spectrum of this tract, we feel justified in concluding that the tract does contain some thick fibres.

It is notable that so many ascending fibres were seen in our material. For the lesions involved the cortico-spinal tract only partially, and the survival periods were so long that much of the terminal parts of the fibres must have completely degenerated and disappeared. Ideal material for working out the detailed anatomy of the ascending fibres within the cortico-spinal tract would be cases in which lesions involved the entire area of the tract and where the survival periods were between five and 40 days.

\section{Conclusions}

The facts set out above lead us to the view that the degenerating fibres under discussion represent a true spino-cerebral tract. Supporting evidence comes from the work of Brodal and Walberg (1952). They demonstrated in cats degeneration of fibres arising in the cord and running to the cerebral cortex. The boutons terminaux around the cortical cells show that this is Wallerian degeneration in a spino-cortical tract. Further supporting evidence comes from Brodal and Kaada (1953). They picked up changes in potential difference from the cat's medullary pyramids, following stimulation of cutaneous, muscular, and mixed peripheral nerves in all limbs. Their findings indicated that the ascending fibres at the level of the pyramids are diffusely scattered throughout the area of the cortico-spinal tract. The majority of the ascending fibres appear to be crossed. As, then, there is such a tract in the cat it is reasonable to expect that the fibres we have described do form a spino-cortical tract in man. We therefore interpret our evidence as indicating that this is a true ascending tract, arising mainly in the cervical segments and running to the cerebral cortex. If this work is eventually confirmed by others it will be established that there is a direct pathway from the spinal cord to the cerebral cortex capable of conducting impulses at great speed. This would be the only direct pathway known not having any synapses between the cord and the cerebral cortex.

\section{A NOTE ON RETROGRADE DEGENERATION}

Forty years after Türck's first description (1851) of the cortico-spinal tract, Sottas (1893), Gombault, and Philippe (1894), and Raymond (1894) described this ascending degeneration within the area of the tract. During these years the idea of a descending cortico-spinal tract had become so firmly fixed that neurologists assumed that degeneration in this area necessarily involved descending cortico-spinal fibres, and therefore must be an example of retrograde degeneration. It came about that the degeneration in the ascending fibres of the lateral cortico-spinal tract area-which Brodal and Walberg (1952) and Brodal and Kaada (1953), and ourselves consider to be a spino-cortical tract-was taken as the typical example of retrograde degeneration. The differences which these earlier workers found in the degeneration of these ascending fibres and that of known descending tracts are due to features of this particular case. For example, the fact that degeneration in the area of the cortico-spinal tract cranial to the lesion was scattered throughout the region of the tract was taken as a main point of differentiation between retrograde degeneration and Wallerian degeneration, for it was claimed that in the latter the entire tract degenerates. However, if these are ascending fibres scattered diffusely among the descending corticospinal fibres, it is clear that when only the former 
degenerate the picture is bound to be one of scattered degeneration. The concept of retrograde degeneration soon became universally accepted, and yet the basic example of this kind of degeneration is now seen to be based on a wrong interpretation of the evidence.

This subject of retrograde degeneration is so important that we include here this note devoted to it.

Waller (1850) thought that the process of degeneration occurs only in the peripheral separated portion of a nerve. Subsequent workers, however, found changes in the central end of a divided nerve. Klippel and Durante (1895) reported their observations on man and experimental animals and reviewed the subject of degeneration in a series of five papers. They recorded retrograde degeneration (dégénérescence cellulipète) in various tracts and peripheral nerves. They stated that retrograde degeneration can be distinguished from Wallerian degeneration (dégénérescence cellulifugé), as in the former the myelin sheath degenerates, often leaving the axons unaffected, whereas in the latter the axons degenerate before the myelin sheaths. When Gombault and Philippe (1894) described the changes they saw in the ascending fibres of the cortico-spinal tract, they believed, in company with other workers of the time, that retrograde degeneration commonly occurs in the central nervous system in man. They noticed that the picture was very like that of Wallerian degeneration, but, searching for differences, they concluded that in this case some of the fibres showed degeneration of the myelin sheaths without degeneration of the axis cylinders, and that in this kind of degeneration there were no normal fibres immediately cranial to the lesion, although they were to be found in increasing number as the tract was followed cranially.

In the subsequent years many other workers investigated the question of retrograde degeneration ; the majority of them could not find or produce this kind of degeneration. Nevertheless, Ramsay Hunt (1904) wrote of this degeneration in detail as follows :-

" The retrograde atrophy of the pyramidal tracts at the first glance resembles the usual descending degenerations. On closer scrutiny certain differences become apparent. The sclerosis is less compact, and contains in its meshes innumerable minute medullated nerve fibres, with attenuated myelin sheaths. ... The axis cylinders are well preserved, although slightly swollen and staining faintly. The retrograde process extends, as a rule, only a few inches above the lesion, and in none of the recorded cases could be followed above the decussation of the pyramids. It is also interesting to note that the pathological changes in the nerve fibres are in a more active stage at the upper limit of the atrophy, i.e., furthest removed from the initial lesion. . . . I can find no record of any case in which the atrophy was apparent before the lapse of two years from the date of the lesion."

In 1906 van Gehuchten reported the results of an experimental study on the rabbit. He found that when the facial or hypoglossal nerve was cut no changes occurred in the fibre central to the cut, but when the nerves were pulled (arrachés) then degeneration indistinguishable from the Wallerian type occurred in the central ends of the fibres. In this case, the degeneration started from the cell body, and was actually an example of Wallerian degeneration, for it was the consequence of the gross changes taking place in the cell body resulting from the tearing of the fibre. Van Gehuchten called this degeneration " dégénérescence Wallerienne indirecte". He considered that the cells of origin of certain nerves and of certain tracts in the central nervous system died when their axons were cut, and consequently the axons of these cells degenerated from the cell-body cellulifugally. The instances he mentioned of this form of degeneration were the rubro-spinal, the reticulo-spinal, the vestibulo-spinal tracts, and the middle cerebellar peduncle.

All these workers used the Marchi method. No better method has yet been found for showing degenerating myelinated fibres; but, as is well known, it tends to produce staining artefacts, and, it must be added, with the techniques used by the workers of those times, these were common. Such artefacts cannot always be easily distinguished from the true staining of degenerating myelin sheaths. There is no doubt that this was not fully realized by some of these earlier workers, and that some of their reported examples of degeneration are in fact examples of the artefacts of the Marchi method. It is unfortunate in this context that few of the early workers could illustrate their papers by photographs, which would have made possible a reassessment of the significance of their findings in the light of later knowledge.

Cajal (1928) in his detailed studies of degeneration processes shows fibres degenerating back to the nearest collateral. He wrote: "The maintenance of the connexion, and therefore the continued functioning, of a collateral, constitutes a hindrance to traumatic degeneration."

Lance (1954) studied retrograde degeneration in the cortico-spinal axons of the cat, following lesions made in the medulla. He came to the conclusion that " an injured pyramidal axon degenerates back to the last node of Ranvier" within some weeks of the injury, and that in the subsequent months it degenerates back to the last collateral. He added 
that " the possibility that some descending pyramidal fibres undergo complete retrograde degeneration back to the cortex cannot be excluded ". But as he did not examine material cranial to the pons, evidence for the actual occurrence of such degeneration was not available.

In considering the work on direct retrograde degeneration, it seems to us that two different forms of this degeneration have been described. There is degeneration throughout the length of a fibre, total degeneration, and there is degeneration present for only a few millimetres central to a lesion, local degeneration. In our opinion, satisfactory reports of the local form of retrograde degeneration have been given, but we have found no reports of total degeneration giving adequate distinctive features which justify one in believing that this total retrograde degeneration differs from true Wallerian degeneration. We therefore doubt whether its existence has been proved.

With regard to the total indirect Wallerian degeneration described by van Gehuchten, the changes spread from the cell-bodies peripherally and not from the periphery centrally, as they do in Wallerian degeneration. Thus, if the entire tract were examined the majority of degenerating fibres would be found near the cells of origin - the contrary to what is seen in true Wallerian degeneration or in direct retrograde degeneration.

As this whole question of retrograde degeneration became important in our studies of tract degenerations, we examined in detail many hundreds of sections from our series of 43 cases. These cases are particularly suitable for investigations of degeneration, as the length of time between the lesion and death is accurately known, the lesion in the cord being a surgical one. We also examined sections of spinal cords from many patients who had at the time of death heart failure, uraemia, alcoholism, cancer, pulmonary tuberculosis, and Parkinsonism.

From this material we conclude that retrograde degeneration occurs in the local form but not in the total form affecting an entire tract. Although we have occasionally seen a few fibres degenerating for a few centimetres in a direction which could be considered as cellulipetal, we cannot accept such changes as adequate evidence of retrograde degeneration. The reasons for this are as follows. First, we have never been able to trace such degeneration back to the cell body, even when the relevant cell bodies have shown gross changes, amounting sometimes to loss of many cells in some nuclei. Secondly, in the spinal cord tracts overlap and intermingle; in any tract there may be a few fibres belonging to another system running in the reverse direction from the main mass of fibres. Thirdly, in view of the nature of the Marchi method, the presence of only a few black bodies central to a lesion needs to be interpreted with great caution. Only when such bodies occur constantly in a certain distribution would we be ready to regard them as significant. Considering these facts, we have never seen any evidence of total retrograde degeneration throughout the length of a tract.

\section{Summary}

Ascending fibres within the region of the corticospinal tract are described in man.

These fibres, intermingled with those of the cortico-spinal tract, run through the pyramidal decussation into the pons. In the cerebral peduncle and internal capsule they lie posterior to the cortico-spinal tract in the region occupied by the temporo-pontine fibres.

In this material most of these fibres were found to be arising in the upper cervical segments of the cord.

It is concluded that these fibres form a spinocortical tract.

The subject of retrograde degeneration, which is relevant to the degeneration in this tract, is discussed,,$\stackrel{\mathbb{Q}}{\Omega}$ and some observations on its non-occurrence areo reported.

We wish to thank Dr. E. A. Carmichael for his helpfulo constructive criticism, Mr. Wylie McKissock who throughout this work, has cooperated in giving us most valuable material, Mr. Peter Sharp and Mrs. Vera Mills for their excellent technical work, and Mr. J. A. Mills for his help in photography.

\section{REFERENCES}

Bing, R. (1905). Arch. Psychiat. Nervenkr., 39, 74.

(1907). Die Bedeutung der spino-cerebellaren Systeme. Bergmann, Wiesbaden.

Brodal, A., and Kaada, B. R. (1953). J. Neurophysiol., 16, 567. and Walberg, F. (1952). Arch. Neurol. Psychiat., Chicago, 68, 755 .

Cajal, S. Ramon y (1928). Degeneration and Regeneration of the Nervous System, trans. R. M. May. Oxford University Press,
London.

Choroschko, W. K. (1909). Mschr. Psychiat. Neurol., 26. 534.

Dejerine, J., and Sottas, J. (1896). Arch. Physiol, norm. path.

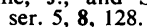

Dercum, F. X., and Spiller, W. G. (1896). Amer. J. med. Sci., 112,672 .'

Egger, F. (1895). Arch. Psychiat. Nervenkr., 27, 129.

Gehuchten, A. van (1906). Anatomie du Système Nerveux de l'Homme, 4th ed. Librairie Universitaire, Louvain.

Gombault, A., and Philippe, - . (1894). Arch. Méd. exp., 6, 365.

Hunt, J. R. (1904). J. nerv. ment. Dis., 31, 504

Klippel, M., and Durante, G. (1895). Rev. Med., Paris, 15, I, 142, 343,574 and 655 .

Lance, J. W. (1954). Brain, 77, 314.

Petrén, K. (1903). Neurol. Zbl., 22, 450.

Raymond, F. (1894). Arch. Neurol., Paris, 27, 112

Sottas, J. (1893). C. R. Soc. Biol., Paris, ser. 9, 5, 925.

Spiller, W. G. (1898). Bull. Johns Hopk. Hosp., 9, 125.

Stewart, P. (1901). Brain, 24, 222.

Tower, S. S. (1944). In P. C. Bucy's The Precentral Motor Cortex. Univ. Illinois Press, Urbana.

Türck, L. (1857). S. B. Akad. Wiss Wien., 6, 288.

Waller, A. (1850). Philos. Trans. B., 140, 423.
Worotynski, B. (1897). Neurol. Zbl., 16, 1094. 